\title{
EL CONSUMO DE CAMÉLIDOS (VICUGNA VICUGNA) EN UN SITIO CEREMONIAL DE LA ALTA CORDILLERA ANDINA (DEPARTAMENTO TINOGASTA, CATAMARCA, ARGENTINA)
}

\author{
CAMELID CONSUMPTION (VICUGNA VICUGNA) FROM A CEREMONIAL SITE \\ IN THE HIGH ANDES (TINOGASTA DEPARTMENT, CATAMARCA, ARGENTINA)
}

\author{
Norma Ratto ${ }^{1}$ y Mariana De Nigris ${ }^{2}$
}

\begin{abstract}
El propósito de este trabajo es discutir a través de los resultados de los estudios zooarqueológicos las interpretaciones realizadas acerca del sitio de altura Fiambalá 1 (5.000 msm, Departamento Tinogasta, Catamarca) para el período Formativo (ca. 530-720 d.C.). El sitio fue considerado como ceremonial con ocupaciones temporales u ocasionales que se enmarcarían dentro del culto a los volcanes. Los materiales óseos fueron recuperados solamente en el Recinto $\mathrm{N}^{\mathrm{o}} 1$ y los análisis efectuados señalan el consumo de ciertas unidades anatómicas de una única especie -vicuña (Vicugna vicugna)- que habría sido obtenida en las vegas de altura ubicadas en pisos inferiores $(4.000 \mathrm{msm})$. Los elementos óseos identificados proceden en su mayoría del esqueleto apendicular y se asocian con el aprovechamiento de la médula ósea, rica en grasas, que le permitiría a los grupos humanos hacer frente a las condiciones climáticas hostiles de emplazamiento del sitio. Además de la escasa diversidad de especies representadas y de la baja densidad de restos óseos recuperados, la ausencia de huesos con grados de termoalteración avanzados nos indica también que nos encontramos frente a un evento de consumo desarrollado en un lapso acotado.
\end{abstract}

Palabras claves: zooarqueología, sitios de altura ceremoniales, período Formativo, Catamarca.

This paper discusses current interpretations of the high altitude Andean site of Fiambala (5,000 masl, Tinogasta Department, Catamarca), which dates to the Formative Period (ca. 530-720 AD), based on zooarchaeological data. The site was originally considered to be ceremonial with temporary or occasional occupations related to the cult of volcanoes. Skeletal remains were only recovered from Structure 1 and zooarchaeological analysis shows the consumption of certain anatomical units coming from a single camelid individual identified as a vicuña (Vicugna vicugna) that was probably obtained from the vegas located in the lower ranges of the Andes (4,000 masl). Most of identified bone elements are limb bones associated with obtaining the fat-rich marrow, an activity that helped allow human groups to face the hostile weather conditions at this site's location. In addition to low species diversity and a low density of bone remains, the absence of bone remains with evidence of high termoalteration also suggests a discrete and brief consumption event.

Key words: Zooarchaeology, high altitude ceremonial sites, Formative Period, Catamarca.

En este trabajo damos a conocer los resultados del análisis del registro arqueofaunístico recuperado del sitio a cielo abierto con estructuras denominado Fiambalá 1 (5.000 msm, Dpto. Tinogasta, Catamarca) que da cuenta del consumo de camélidos sudamericanos silvestres en pisos de altura de alta cordillera por parte de sociedades formativas (ca. 530-720 d.C.). El sitio se emplaza en la confluencia entre los volcanes Incahuasi Grande e Incahuasi Chico en plena Cordillera de los Andes, específicamente en el área conocida como "Los Seismiles" ya que se encuentran las montañas y volcanes más altos de América (Figura 1). Una de las características del sitio Fiambalá 1 es que es multicomponente con ocupaciones que remiten a tiempos de las primeras sociedades agropastoriles y la ocupación incaica en la región. Sin embargo, el consumo de camélidos sólo está registrado en el primero de los momentos señalados en asociación directa con el área de limpieza de un fogón registrado en uno de los recintos del sitio.

Cabe destacar que el sitio fue considerado como parte integrante de las actividades ceremoniales realizadas en las altas cumbres andinas desde tiempos de las primeras sociedades productivas hasta la ocupación incaica que incluyeron rituales apaciguadores a los volcanes (Ratto y Orgaz 2009). Por lo tanto, consideramos que el análisis

\footnotetext{
1 Museo Etnográfico Juan B. Ambrosetti, Facultad de Filosofía y Letras, Universidad de Buenos Aires, Moreno 350 (1091), Ciudad Autónoma de Buenos Aires, Argentina. nratto@filo.uba.ar

2 CONICET-Instituto Nacional de Pensamiento Latinoamericano, 3 de Febrero 1370 (1426) Ciudad Autónoma de Buenos Aires, Argentina. mariandenigris@yahoo.com.ar
} 


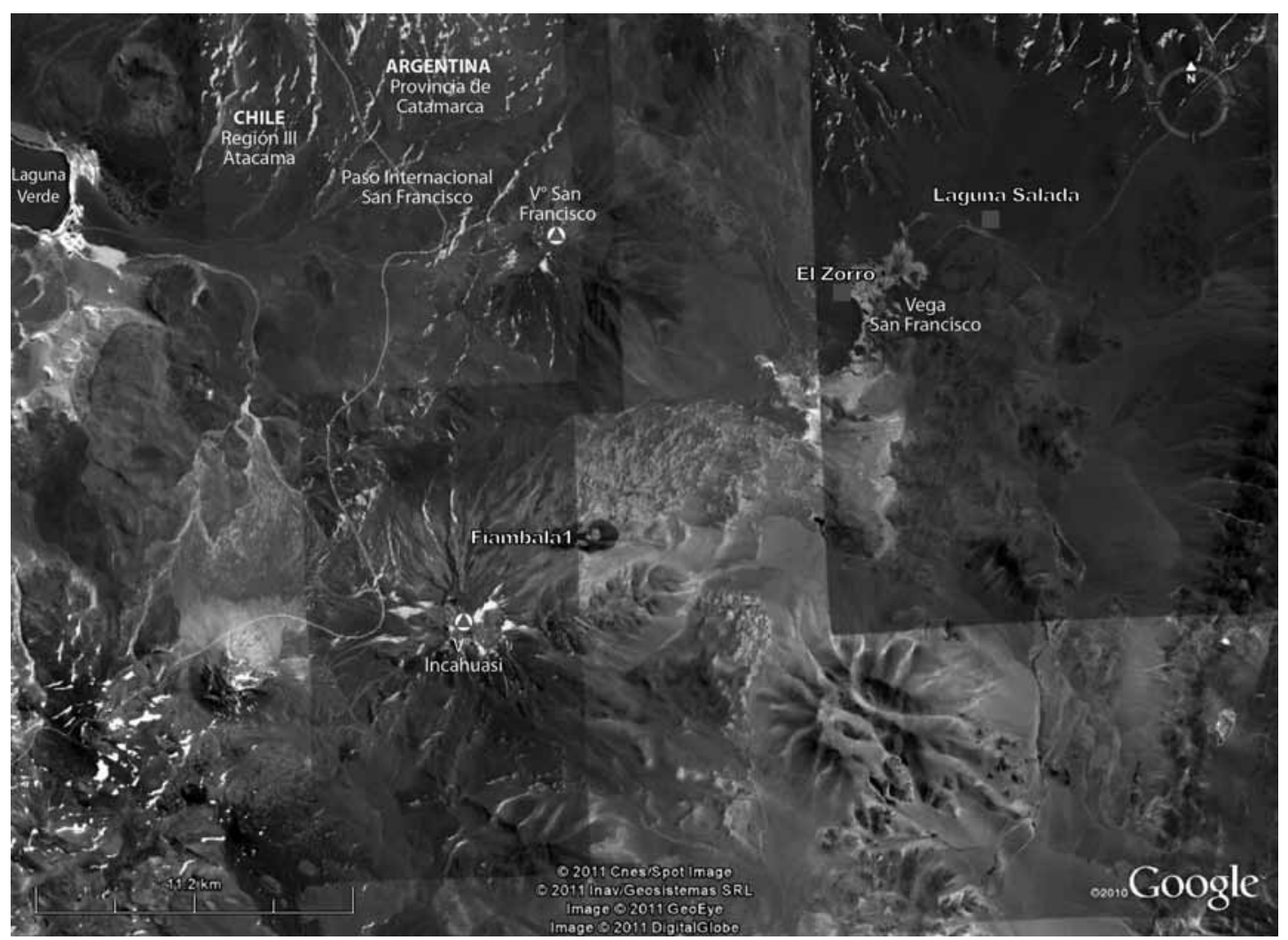

Figura 1. Localización de los sitios Fiambalá 1 (5.000 msm), cerca del límite internacional argentino-chileno, El Zorro (4.050 msm) y Laguna Salada (4.000 msm).

Location of Fiambalá 1 (5,000 masl), near the Argentine-Chilean frontier, El Zorro (4,050 masl) and Laguna Salada (4,000 masl).

del registro arqueofaunístico aporta una nueva línea de evidencia para corroborar o rechazar la hipótesis de la ocupación temporal u ocasional del sitio en el marco de actividades ceremoniales relacionadas con el culto a los volcanes. En este contexto, el análisis específico contempla la identificación anatómica y taxonómica de los especímenes óseos, el estudio del perfil etario, la cuantificación a través de distintas medidas (NR, NISP, MNI, MNE y MAU), la evaluación de la integridad de las muestras (marcas de acción antrópica y natural y el grado de fragmentación).

\section{El Sitio Arqueológico Fiambalá 1}

Hasta el registro, documentación y análisis del sitio Fiambalá 1 (5.000 msm) no se tenía conocimiento en el NOA de la existencia de espacios arquitectónicos multicomponentes con testimonio tanto de sociedades Formativas (ca 500-700 d.C.) como Incaicas (ca. 1.400-1.460 d.C.).
El sitio se emplaza dentro de la provincia Altoandina que pertenece al Dominio Andino-Patagónico de la Región Neotropical, el que se extiende por las altas montañas del oeste del país por encima de los $4.400 \mathrm{msm}$. Los suelos son pedregosos, arenosos, sueltos e inmaduros; el clima de alta montaña es frío y seco, con precipitaciones en forma de nieve o granizo, y la vegetación de gramíneas, líquenes y vegas desaparece a medida que se asciende en las cotas altitudinales (Cabrera 1971). Particularmente, en la cota de emplazamiento del sitio la vegetación es nula, ubicándose al pie del portezuelo definido por las dos cumbres del volcán Incahuasi alcanzando la mayor los $6.638 \mathrm{msm}$. A través de dicho portezuelo es posible realizar el ascenso a la alta cumbre, aunque no es la ruta utilizada en la actualidad por los andinistas.

La cumbre mayor del Incahuasi tiene forma de cono obtuso emplazándose en el límite argentino-chileno, siendo además extremo de una larga cadena de otros gigantes volcánicos tales como San Francisco, 
El Fraile, El Muerto, Ojos del Salado, Cerro Solo y Tres Cruces de la Cordillera de los Andes. Cabe destacar que la cima del volcán guarda los restos de un sitio ceremonial o santuario de altura (Bulacio 1998; Ratto y Orgaz 2009). Parte de los objetos recuperados en el santuario estuvieron expuestos en el Banco Nación de la ciudad de San Fernando del Valle de Catamarca (Orgaz y Morales 1999).

El arreglo espacial arquitectónico está compuesto por cinco estructuras pircadas de forma circular y/o subcircular. Una de ellas se localiza a unos siete metros del conjunto principal, mientras que las cuatro restantes comparten muros y presentan tamaños diferenciales que oscilan entre dos y seis metros de diámetro aproximadamente. La estructura de mayor tamaño presenta un muro divisor interno, habiendo sido construido todo el conjunto arquitectónico con rocas volcánicas locales tipo dacíticas y andesíticas (Figura 2).

Actualmente el sitio se encuentra colmatado por piedra pómez redepositada por el viento, pero fue fundado también sobre material de origen pumíceo. La excavación reportó diferente tipo de evidencia artefactual y ecofactual, posibilitando esta última la realización de análisis geoarqueológicos (Kligmann y Ratto 2009). En los tres recintos excavados (Figura 2) se hallaron techos colapsados, formados por poaceas (gramíneas) unidas por cordeles de las mismas materias primas (aff. Deyeuxia sp., Festuca aff. scirpifolia y aff. Festuca sp.). Los paneles del techo fueron soportados por troncos de algarrobo

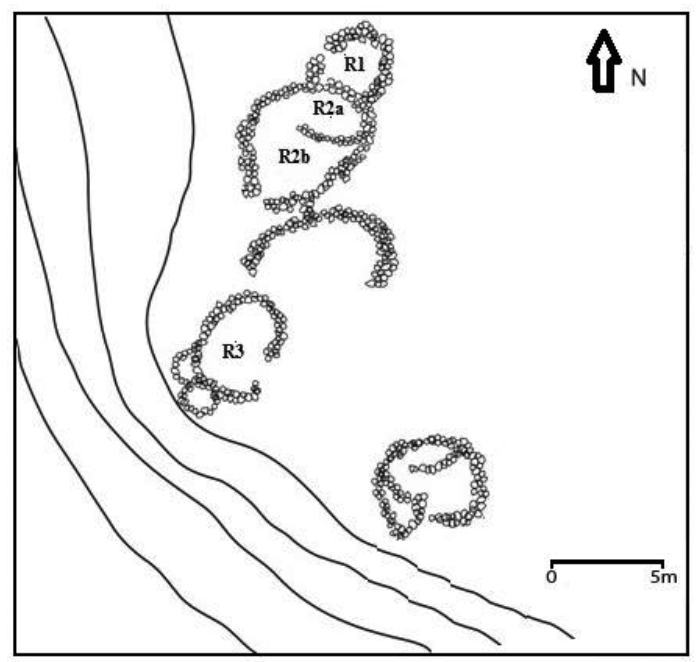

Figura 2. Planimetría y señalización de los recintos intervenidos en el sitio Fiambalá 1 (5.000 msm).

Map of the investigated structures of Fiambalá 1 (5,000 masl).
(Prosopis spp) a modo de viga de sostén (Ratto et al. 2010). En una de las estructuras (Recinto $\mathrm{N}^{\circ} 1$ en Figura 2) fue documentado y registrado un fogón tipo plano rodeado por rocas y asociado a un área de limpieza con presencia de carbones y otros macrorrestos vegetales quemados como chañar (Geoffroea decorticans). De este sector proviene el único registro arqueofaunístico recuperado en el sitio y analizado en este trabajo.

Es interesante que en asociación con el fogón se recuperara una roca volcánica ácida, de tamaño mediano, con pigmento rojo adherido cuyo análisis por DRX determinó que se trata de hematita. Por su parte, en el Recinto $\mathrm{N}^{\mathrm{0}} 2$, sólo se halló una tecnofactura en materia vegetal en la parte intervenida del sector $b$, interpretada como un "bastón de caminante" (Ratto et al. 2010). La intervención del Recinto $\mathrm{N}^{\circ} 3$ arrojó también resultados estériles en cuanto a evidencia artefactual lítica, cerámica y/o metalúrgica, encontrándose paneles de techo colapsado que apoyan sobre material pumíceo y por debajo se registró un área carbonosa quemada con presencia de fragmentos de carbón de tamaños pequeños.

En la actualidad se cuenta con cinco fechados radiocarbónicos. Dos de estos corresponden a carbones registrados en el fogón del Recinto $\mathrm{N}^{\circ} 1$ y el área carbonosa del $\mathrm{N}^{\mathrm{o}} 3$. Los tres restantes provienen de gramíneas de los techos colapsados de los Recintos $\mathrm{N}^{\mathrm{o}} 2$ y No 3 (Tabla 1 y Figura 3 ).

Los estudios geoarqueológicos indican claramente la existencia de dos momentos de ocupación

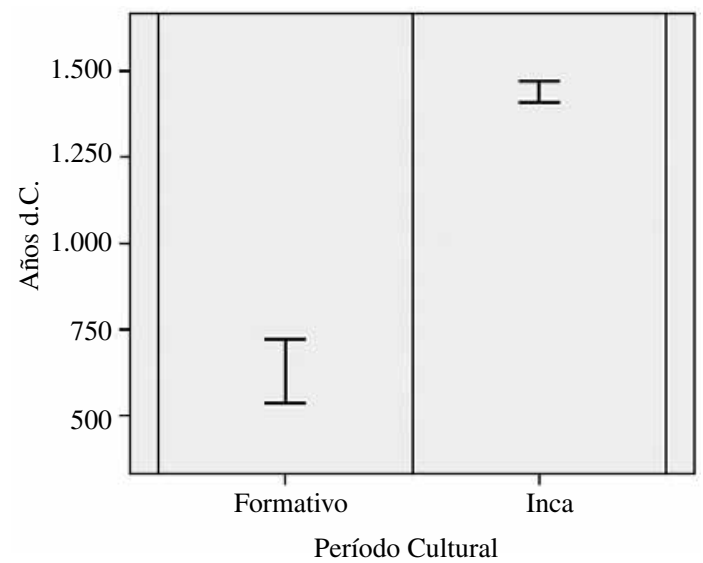

Figura 3. Boxplot con los fechados radiocarbónicos (1 sigma) del sitio multicomponente Fiambalá 1.

Radiocarbon date boxplot (1 sigma) from the multicomponent site of Fiambalá 1. 
Tabla 1. Fechados radiocarbónicos del sitio Fiambalá 1.

Radiocarbon dates from the Fiambalá 1 site.

\begin{tabular}{ccccc}
\hline Laboratorio & Material & $\begin{array}{c}\text { Años radiocarbónicos } \\
\text { (a.p.) }\end{array}$ & $\begin{array}{c}\text { Fechado calibrado } \\
\text { (cal d.C.) 1 sigma }\end{array}$ & Período Cultural \\
\hline AA81740 & Carbón en área carbonosa del Recinto No 3 (M132) & $1.499 \pm 51$ & $539 \pm 65$ & Formativo \\
AA69976 & Carbón procedente del fogón del Recinto No 1 (M46) & $1.294 \pm 33$ & $715 \pm 39$ & Formativo \\
AA81739 & Gramínea de techo colapsado del Recinto No 3 (M125) & $504 \pm 36$ & $1.415 \pm 14$ & Inca \\
AA81741 & Gramínea de techo colapsado del Recinto No 3 (M129) & $458 \pm 49$ & $1.450 \pm 32$ & Inca \\
AA69977 & Gramínea de techo colapsado del Recinto No 2 (M66) & $465 \pm 34$ & $1.432 \pm 12$ & Inca \\
\hline
\end{tabular}

de muy baja potencia sedimentaria (Kligmann y Ratto 2009). Estos fueron identificados a través de la modificación antrópica de sedimentos procedentes de las áreas intervenidas con excepción del Recinto $\mathrm{N}^{\circ} 3$ que se encuentra en proceso de análisis. A saber:

(a) El primer componente corresponde al fogón del Recinto $\mathrm{N}^{\mathrm{o}} 1$ con asociación del registro arqueofaunístico y otros macrorrestos vegetales, correspondiendo por su fechado a tiempos del Formativo, al igual que los carbones del área quemada registrada en el Recinto $\mathrm{N}^{\circ} 3$. En el primer caso el conjunto indica un uso del espacio moderadamente más intensivo que el registrado para tiempos posteriores, postulándose la realización de actividades rituales dentro del sitio y/o en sus inmediaciones debido a la recuperación de la roca con pigmento rojo adherido (Kligmann y Ratto 2009; Ratto y Orgaz 2009). Los paneles de techo colapsado situados por arriba del fogón tienen fechados en curso, mientras que los del Recinto $\mathrm{N}^{\circ} 3$ lo sitúan en tiempos incaicos (ver más adelante).

(b) El segundo componente está representado por los Recintos $\mathrm{N}^{\circ} 2$ y $\mathrm{N}^{\circ} 3$. En el primero la única evidencia de ocupación proviene de la existencia del techo colapsado sin otro tipo de macrovestigios artefactuales y/o ecofactuales, con excepción del "bastón de caminante", que de acuerdo al fechado obtenido corresponde a momentos de la ocupación incaica en la región. El contexto da cuenta de una ocupación muy efímera postulándose la realización de actividades de alcance simbólico-ceremonial que no conllevaron generación de residuos (Kligmann y Ratto 2009; Ratto y Orgaz 2010). Por su parte, los paneles de techo colapsado del Recinto $\mathrm{N}^{\circ} 3$ arrojaron fechas incaicas determinándose que los paneles del derrumbe del techo están intercalados por eventos de depositación de piedra pómez de origen eólico.

Finalmente, el aporte brindado por los estudios paleoambientales, sobre la base del análisis de sedimentos lacustres con complemento de otros proxies, permitió delinear un escenario físico puneño que no guarda ninguna relación con el actual. Este aspecto fue fundamental para recrear diferentes escenarios físicos a lo largo del Holoceno caracterizados por períodos de mayor y menor humedad relativa. Los resultados refuerzan la idea de los grandes cambios en la disponibilidad hídrica en la Puna que incidieron directamente en la dinámica de las vegas de altura como concentradores de nutrientes, resultando en ciclos de expansión y retracción de las mismas (Ratto 2007; Valero et al. 2007). Estos cambios dan cuenta de períodos secos que fluctuaron con otros de mayor humedad relativa a partir de comienzos de la era, siendo este el escenario con el que interactuaron las sociedades agropastoriles del 500-700 d.C.

\section{Metodología del Análisis Zooarqueológico}

El análisis de las muestras óseas recuperadas se centró en: la identificación anatómica y taxonómica de los especímenes que involucró el uso de criterios osteométricos y de correspondencias anatómicas; la consideración de los perfiles anatómicos y etarios, y el registro de modificaciones tanto antrópicas como naturales.

Varias medidas fueron utilizadas en relación con el primer punto. El NR o Número de Restos tiene en cuenta la totalidad de especímenes óseos recuperados durante las excavaciones que hayan podido ser identificados o no. Para aquellos que pudieron ser determinados hemos utilizado dos medidas: el NISP o Número de Especímenes Identificados por Taxón (Lyman 2008) y el MNI o Número Mínimo de Individuos (Lyman 2008). 
El NISP se calculó sumando tanto los huesos enteros como los fragmentados para cada una de las especies determinadas, así como también para aquellas categorías taxonómicas de carácter más incluyente, como por ejemplo Artiodactyla. El MNI se obtuvo teniendo en cuenta la lateralidad y el estado de fusión de los especímenes.

Las peculiaridades de las muestras óseas recuperadas en el sitio -excelente estado de preservación $\mathrm{y}$ alta integridad- nos han permitido implementar el uso de correspondencias anatómicas -unión intermembral y el remontaje mecánico (Messineo y Kaufmann 2001) - como herramientas metodológicas para afinar la cuantificación de la abundancia taxonómica y anatómica. La unión intermembral es cuando se acoplan unidades que articulan entre sí, mientras que el remontaje mecánico se refiere a la unión de especímenes fracturados que originalmente pertenecían al mismo elemento óseo.

Asimismo, hemos utilizado una serie de criterios osteométricos para poder discriminar entre distintas especies de camélidos. Dado lo exiguo de la muestra analizada muy pocos especímenes pudieron ser medidos, solamente hemos utilizado las falanges 1 aplicando la técnica bivariada que consiste en la consideración de dos dimensiones simultáneamente que pueden expresarse mediante el uso de diagramas de dispersión. Para su elaboración hemos aplicado los criterios propuestos para camélidos por Kent (1982). Se consideraron el ancho máximo y el espesor de la superficie articular proximal -1FA2 y 1 FA $3 \mathrm{~K}$ respectivamente-. Los valores de referencia actuales proceden de Elkin (1996) e Izeta et al. (2009). La diferenciación entre falanges delanteras y traseras se realizó siguiendo las pautas propuestas por Cartajena (2009).

En el caso de los camélidos hemos considerado la representación anatómica de las diversas partes esqueletarias a través del MNE o Número Mínimo de Elementos (Binford 1984). Dada la fragmentación de las muestras consideradas fue necesario recomponer los diferentes elementos óseos, teniendo en cuenta las diversas zonas diagnósticas que los conforman (Mengoni Goñalons 1999) y los reensamblajes mecánicos realizados. Se obtuvo luego el Número Mínimo de Unidades Anatómicas o MAU, para permitir la comparación, el cual se obtiene de la razón entre el MNE y el número de veces que determinado elemento aparece representado en el esqueleto (Binford 1984).
Se valoró también si los patrones de representación de unidades anatómicas de camélidos obtenidos para el Recinto $\mathrm{N}^{\circ} 1$ eran el resultado de una supervivencia diferencial dependiente de la densidad ósea (Lyman 1994). Para evaluar esa posibilidad correlacionamos el \%MAU calculado para cada uno de los elementos presentes con los valores de densidad ósea obtenidos por Elkin (1995).

La estimación de la edad sobre la base de la fusión ósea fue otro aspecto tenido en cuenta. Para ello, se siguieron los criterios propuestos por De Nigris (2004) considerando aquellos elementos que tienen un cronograma de fusión más acotado temporalmente y se agregaron algunas consideraciones de Kaufmann (2009) especialmente en relación con las vértebras.

En cuanto a las modificaciones que presentaban los restos óseos analizados se consideraron aquellas que son resultado de la actividad humana entre las cuales fueron registradas fundamentalmente huellas de corte y percusión -hoyos y estrías(Lyman 1994), junto con negativos de impactos que pueden ser asociados a la fractura intencional de los huesos (Capaldo y Blumenschine 1994). Por otra parte, se efectuó también una evaluación del estado del hueso al momento de producirse la fractura-fresco o seco-(Gifford-González 1989). Durante el análisis se prestó especial atención a las transformaciones que podían haber sido ocasionadas por otro tipo de agentes y procesos, tales como la acción de carnívoros, roedores, la meteorización y las marcas de raíces, aunque ninguna de ellas pudo ser identificada.

Otro aspecto considerado fue la presencia de alteraciones producidas por la acción térmica. Se consideró el color que exhibían los especímenes óseos estableciéndose cinco estadios progresivos diferentes: no quemado o crudo (blanquecino/amarillento); quemado incipiente (amarillo); quemado avanzado (rojizo/marrón); carbonizado (negro); y calcinado (gris azulado, blanco, ante) (Mengoni Goñalons 1999).

\section{Resultados del Análisis Zooarqueológico}

La muestra analizada es de carácter reducido si bien incluye todos los especímenes óseos recuperados en el sitio. En la Tabla 2 se muestran los resultados de la identificación taxonómica. En ella se observa la escasa diversidad de especies presentes, siendo los camélidos los únicos determinados. Esta situación 
Tabla 2. Representación taxonómica del Recinto 1 de Fiambalá (NISP) con y sin ensamblajes mecánicos. Taxonomic Diversity (NISP) in Structure $N^{o} 1$ of Fiambalá 1 with and without mechanical refits.

\begin{tabular}{ccc}
\hline Taxón & $\begin{array}{c}\text { Recinto 1 } \\
\text { (sin remontajes) }\end{array}$ & $\begin{array}{c}\text { Recinto 1 } \\
\text { (con remontajes) }\end{array}$ \\
\hline Artiodactyla & 13 & 3 \\
Camelidae & 38 & 49 \\
Mamíferos indeterminados & 17 & 16 \\
NISP total & 68 & 68 \\
No identificables & 43 & 43 \\
NR Total & 111 & 111 \\
\hline
\end{tabular}

Tabla 3. Representación anatómica de camélidos en el Recinto 1 de Fiambalá. Camelid skeletal profiles in Structure $N^{o} 1$ of Fiambalá 1.

\begin{tabular}{crccc}
\hline Unidad Anatómica & NISP & MNE & MAU & $\%$ MAU \\
\hline Cervicales 3-7 & 7 & 2 & 0,4 & 40 \\
Radioulna: proximal & 3 & 2 & 1 & 100 \\
Radioulna: diáfisis & 8 & 1 & 0,5 & 50 \\
Carpianos & 2 & 2 & 0,1 & 10 \\
Tibia: diáfisis & 2 & 1 & 0,5 & 50 \\
Tarsianos & 3 & 3 & 0,3 & 30 \\
Astrágalo & 3 & 1 & 0,5 & 50 \\
Calcáneo & 1 & 1 & 0,5 & 50 \\
Metapodio: diáfisis & 13 & 2 & 0,5 & 50 \\
Falange 1 & 3 & 2 & 0,2 & 20 \\
Falange 2 & 2 & 2 & 0,2 & 20 \\
Falange 3 & 2 & 2 & 0,2 & 20 \\
NISP total & 49 & & & \\
\hline
\end{tabular}

se hace todavía más evidente cuando se considera la representación taxonómica luego de la realización de remontajes mecánicos (Figura 4), dado que se reduce notoriamente la cantidad de especímenes asignados a Artiodactyla, sugiriendo que la mayoría de los especímenes recuperados pertenecían originalmente a la familia Camelidae cuyo MNI corresponde a un solo individuo (Tabla 3). La categoría mamíferos indeterminados está conformada únicamente por lascas óseas, una de ellas pudo ser reensamblada a un metapodio de camélido.

Los resultados del análisis osteométrico sobre las falanges 1 proximales indican que el tamaño pequeño de los especímenes estudiados es muy semejante a los registrados en vicuñas (Vicugna vicugna) actuales (Figura 5). Ambas falanges poseían exactamente las mismas dimensiones y sus características morfológicas generales confirman también que pertenecían a un mismo individuo.
Asimismo, las uniones intermembrales que han podido efectuarse entre las falanges primeras a terceras indican que todas ellas proceden de un mismo individuo (Figura 6). Un hecho semejante sucede con los carpianos y tarsianos que también articulan entre sí.

Si bien las características generales de la muestra no permiten la medición de otras articulaciones, consideramos que las uniones realizadas, los tamaños generales observados y las características cualitativas son evidencia suficiente para sugerir que los especímenes identificados como camélidos proceden de un único individuo.

La representación anatómica del Recinto $\mathrm{N}^{\mathrm{o}} 1$, tal como evidencia la Tabla 3, es muy sesgada. Un solo elemento óseo-vértebras cervicales-representa al esqueleto axial, mientras que la región apendicular se presenta algo más completa registrándose algunos huesos largos de las patas delantera y 


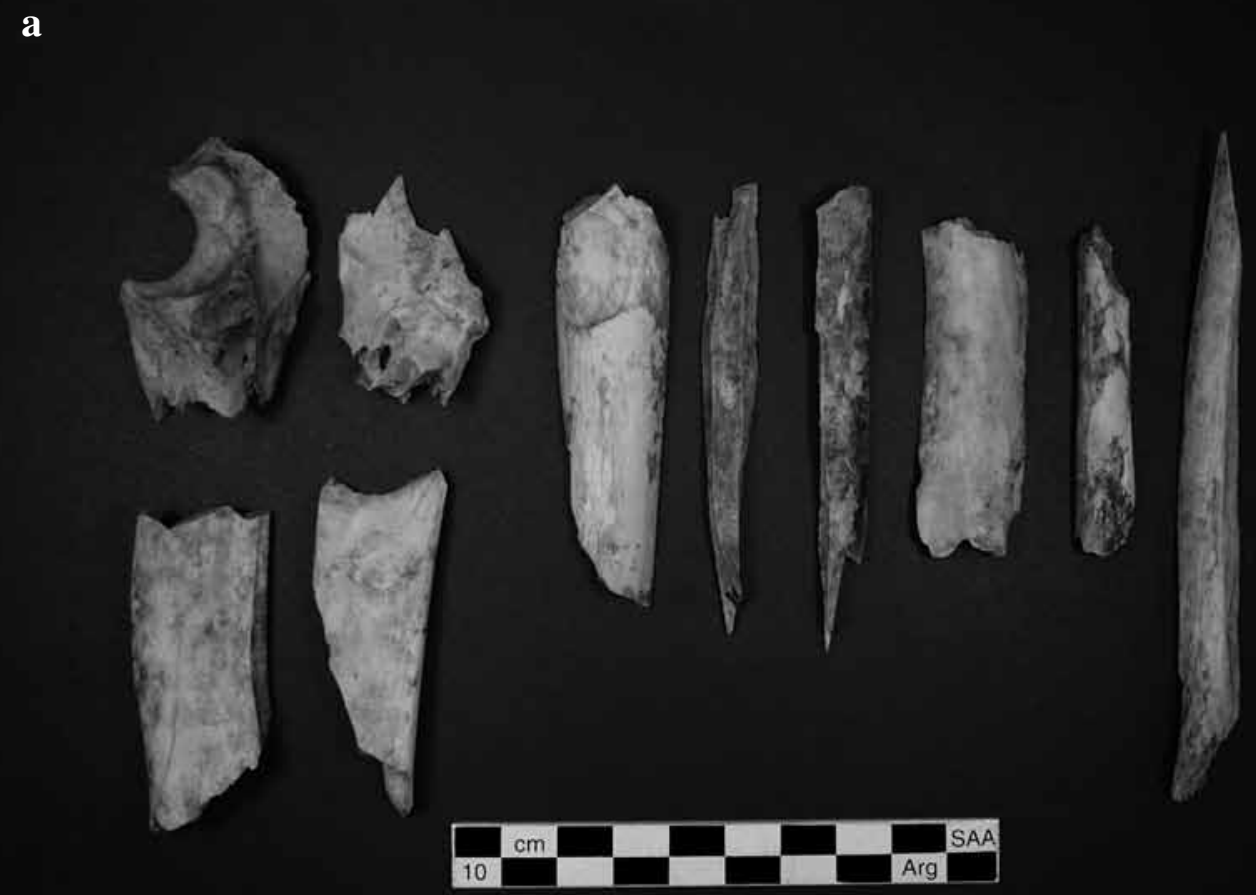

b
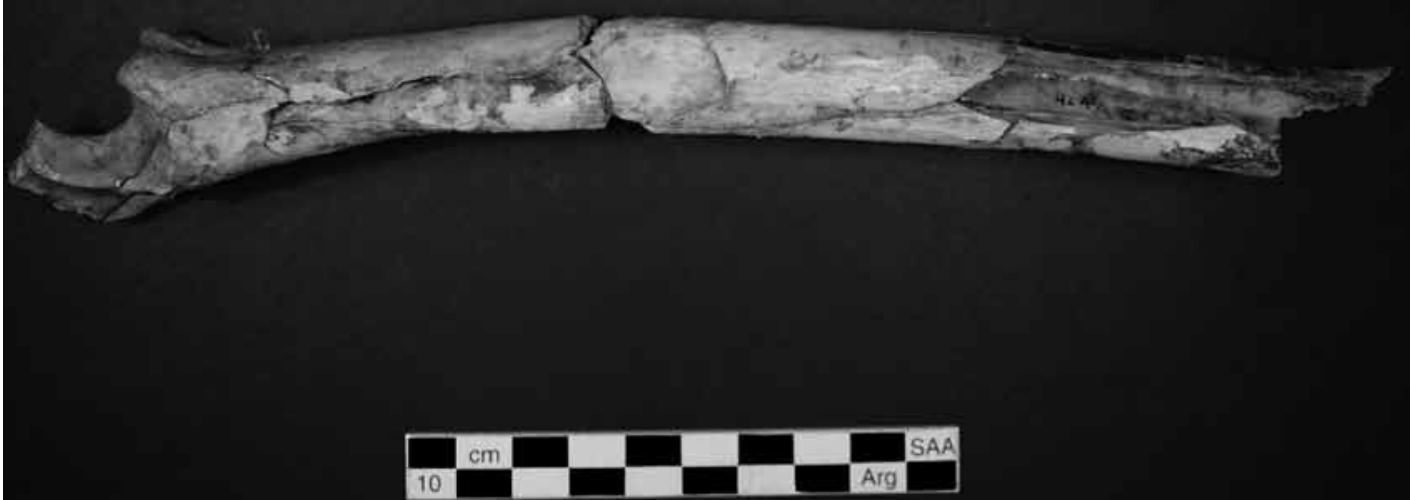

Figura 4. Reensamblajes realizados en un radioulna de camélido posiblemente vicuña. (a) los 10 especímenes y (b) el elemento remontado.

Camelid (vicugna) radioulnae mechanical refits. (a) 10 isolated specimens and (b) refitted element. 
Falange 1 (proximal)

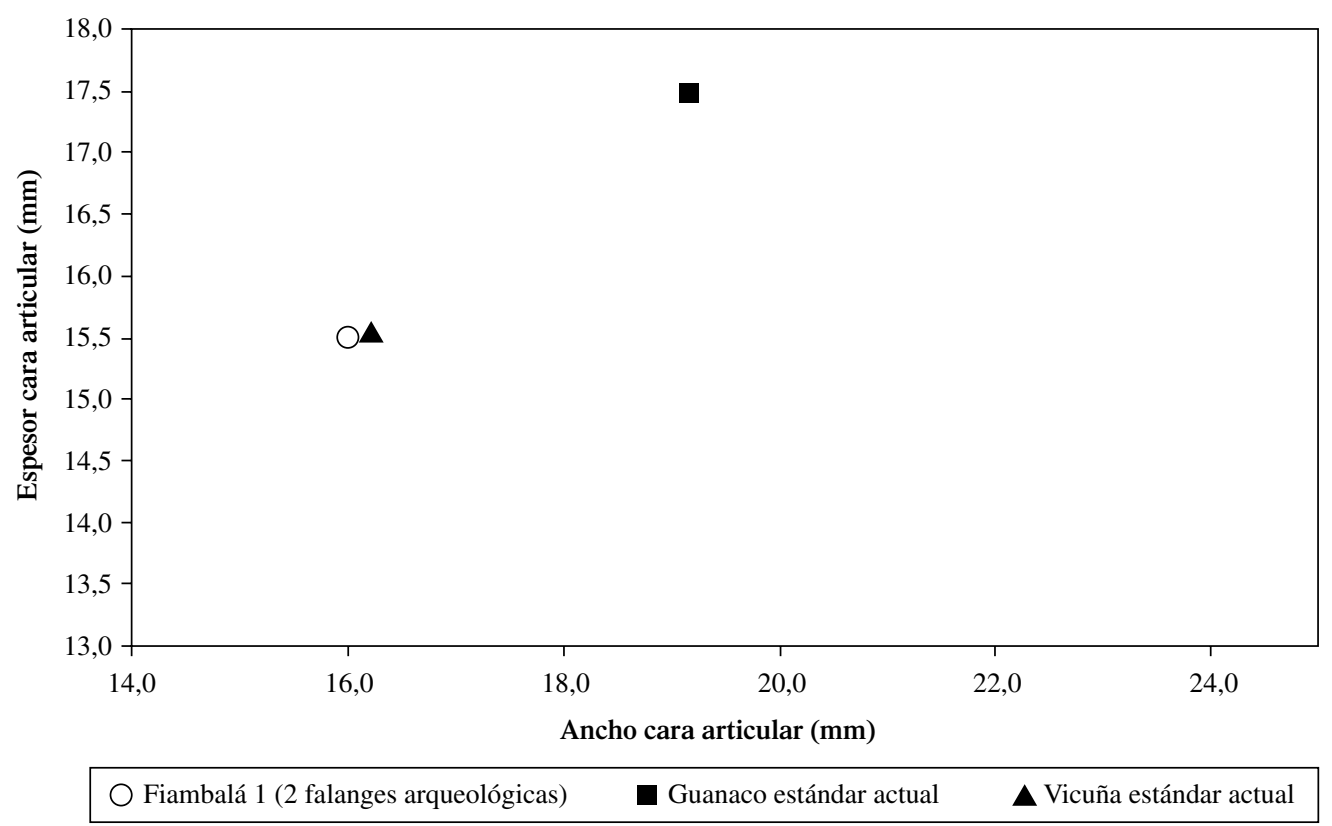

Figura 5. Diagrama de dispersión de las mediciones de las falanges 1 proximales comparando los especímenes actuales (guanaco y vicuña) y arqueológicos del sitio Fiambalá 1 .

Scatter plot of the measurement of proximal phalange 1 comparing extant specimens (guanaco and vicugna) and Fiambalá 1 archaeological specimens.

trasera, carpianos, tarsianos y falanges. El conjunto analizado exhibe de esta forma un patrón de representación anatómica muy particular caracterizado por la ausencia de la cabeza, los costillares y las cinturas y los huesos largos apendiculares más ricos en nutrientes.

Dado lo expuesto, evaluamos si el perfil anatómico registrado podía ser resultado de una preservación diferencial de las diferentes unidades anatómicas. Los resultados obtenidos de la correlación entre la densidad ósea y el \%MAU muestran valores estadísticos moderados-bajos al valor de significación considerado $\left(r_{s}=0,40 p<0,05\right)$. Este guarismo podría estar indicando que la ausencia de ciertas unidades anatómicas podría obedecer a factores densitodependientes. Sin embargo, es interesante observar que la covariación entre el Índice de Utilidad de Carne obtenido por Borrero (1990) para guanaco ${ }^{1}$ y el \%MAU de Fiambalá son estadísticamente significativos con valores medios y negativos $\left(r_{s}=-0,45 p<0,05\right)$. Este hecho se repite cuando confrontamos el volumen de la cavidad medular calculado por Mengoni Goñalons (1996) para la llama y las unidades anatómicas con tuétano del Recinto $\mathrm{N}^{\circ} 1\left(\mathrm{r}_{\mathrm{s}}=-0,35 \mathrm{p}>0,05\right)$. Estos resultados serían consistentes con lo que Thomas y Mayer (1983) han definido como reverse utility strategy, en la cual se registra una gran cantidad de partes anatómicas de bajo rendimiento económico concordante con situaciones de matanza y/o procesamiento inicial o sitios en los que existe una fuerte acción de procesos dependientes de la densidad ósea.

Los resultados de la correlación con la densidad ósea podrían estar señalando la acción de procesos destructivos de carácter postdepositacional. Sin embargo, como veremos más adelante, si bien se han registrado elevados índices de fragmentación producto del consumo de la médula no hay evidencias de acción destructiva por parte de ningún otro agente. Además, el reensamblaje de huesos del conjunto también apunta a señalar que no existe una destrucción de los huesos menos densos in situ sino que consideramos que no formaron parte del conjunto óseo depositado. Cabe resaltar la excelente preservación de los materiales debido a distintos factores, que en gran parte están relacionados con la zona y la cota altitudinal del emplazamiento del sitio. Al respecto, podemos mencionar que no existen agentes tafonómicos como roedores, 


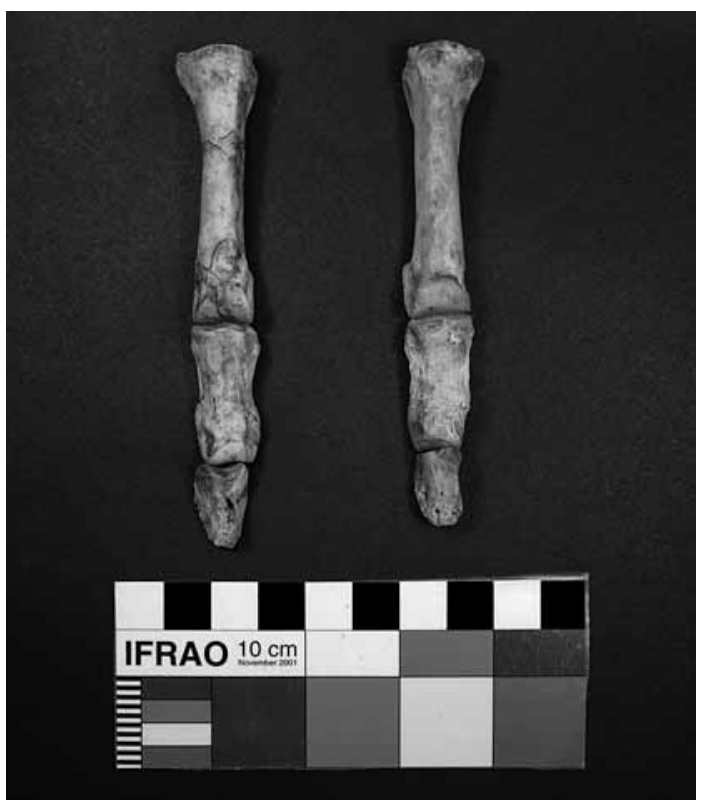

Figura 6. Uniones intermembrales de falanges primeras a terceras de camélido (vicuña).

Intermembranal joints from first to third camelid phalanges (vicugna).

carnívoros, raíces, entre otros, como así también la rápida colmatación de los recintos con material pumíceo de origen eólico, que a su vez se congela durante gran parte del año.

Por otra parte, el estado de fusión de los especímenes solo pudo evaluarse en una vértebra cervical y en las falanges. Todos estos elementos se encontraban soldados indicando que el ejemplar era mayor a 24 meses al momento de su muerte.

Con respecto a las huellas de procesamiento, señalamos que fueron registradas principalmente en el grupo de los camélidos con un predominio de la percusión por sobre el corte $(28,6 \%$ y $14,3 \%$, respectivamente) (Tabla 4). La gran mayoría de las marcas de corte corresponden a desarticulación y unas pocas son interpretadas como descarne. Los porcentajes registrados son consistentes con situaciones de procesamiento inicial de las presas. En la categoría Artiodactyla no se observó ninguna marca y solamente una lasca ósea presentó huellas de percusión -hoyos y estrías- en el conjunto de mamíferos indeterminados.

De lo expuesto, es llamativa la presencia de elevados porcentajes de marcas de percusión indicando que el consumo de médula es una práctica que se desarrolló en este sitio de altura del ambiente cordillerano. Todos los huesos que poseen médula del conjunto se encuentran fracturados a excepción de dos falanges que presentaban malformaciones óseas. La presencia de negativos de impacto, huellas de percusión y el estado fresco de los huesos al momento de fracturarse apuntan a una rotura intensiva a pesar de que las proporciones de médula de los huesos presentes en el conjunto no son demasiado elevadas.

Finalmente, en cuanto a la evidencia de alteraciones térmicas señalamos que alrededor del $60 \%$ de los especímenes (incluidas todas las categorías

Tabla 4. Marcas de procesamiento y negativos de impacto en el Recinto 1, sitio Fiambalá. Processing marks and impact notches in Structure $N^{o} 1$ of Fiambalá 1.

\begin{tabular}{cclll}
\hline Unidad Anatómica & NISP & Corte & Percusión & Negativos \\
\hline Cervicales 3-7 & 7 & $1(14,3 \%)$ & $1(14,3 \%)$ & - \\
Radioulna: proximal & 3 & - & $1(33,3 \%)$ & - \\
Radioulna: diáfisis & 8 & $1(12,5 \%)$ & $2(25 \%)$ & $2(25 \%)$ \\
Carpianos & 2 & - & - & - \\
Tibia: diáfisis & 2 & - & - & $1(50 \%)$ \\
Tarsianos & 3 & $2(66,7 \%)$ & - & - \\
Astrágalo & 3 & $1(33,3 \%)$ & - & $1(33,3 \%)$ \\
Calcáneo & 1 & - & $1(100 \%)$ & - \\
Metapodio: diáfisis & 13 & $1(7,7 \%)$ & $9(69,2 \%)$ & $2(15,4)$ \\
Falange 1 & 3 & $2(66,7 \%)$ & $1(33,3 \%)$ & - \\
Falange 2 & 2 & - & $1(50 \%)$ & - \\
Falange 3 & 2 & - & - & - \\
NISP total & 49 & $7(14,3 \%)$ & $16(28,6 \%)$ & $6(12,25 \%)$ \\
\hline
\end{tabular}


determinadas) presentan algún grado de modificación -quemado incipiente: $57,3 \%$ y quemado avanzado: 2,6\%-; mientras que los restantes se encuentran en estado crudo. No se registraron huesos con grados de termoalteración avanzados como carbonizados o calcinados.

\section{Discusión}

Los resultados del análisis del registro arqueofaunístico del componente Formativo del sitio Fiambalá 1 dan cuenta de un episodio o evento de consumo en condiciones extremas (ca. 700 d.C.). $\mathrm{Al}$ respecto, esta afirmación la sustentamos con los siguientes indicadores:

(a) En la actualidad, el piso altitudinal de $5.000 \mathrm{msm}$ y superiores del área cordillerana no presenta manto de vegetación y consecuentemente la presencia de camélidos es nula, a diferencia de lo que ocurre en pisos inferiores $(4.000 \mathrm{msm})$, pudiendo ser estas condiciones extrapolables al momento de ocupación del sitio (ca. 500700 d.C.). Esto sugiere que el único ejemplar identificado del conjunto óseo analizado representa una presa (vicuña) cazada en las vegas de altura. Al respecto, en los alrededores de estas vegas concentradoras de nutrientes se emplazan otros sitios Formativos como El Zorro y Laguna Salada (Figura 1) cuyos fechados radiocarbónicos son sincrónicos con los del sitio Fiambalá 1 pero sus dimensiones son mucho mayores (Ratto et al. 2002, 2011).

(b) Las unidades anatómicas identificadas en el conjunto tienen una utilidad económica moderada-baja. Al respecto, recordamos que el patrón identificado no responde a problemas de preservación diferencial ligados con la densidad ósea y/o acción de agentes tafonómicos. Por lo tanto, consideramos que las partes dan cuenta de un evento de consumo desarrollado en un tiempo acotado. Asimismo, la ausencia de huesos carbonizados y/o calcinados apunta en esa dirección, debido a que evidencian la falta de manejo de los desechos que sería esperable en situaciones de ocupaciones repetidas en el tiempo.

(c) La elevada fragmentación del conjunto óseo y la presencia de huellas de percusión y negativos de impacto, asociadas con un perfil anatómico dominado por los huesos apendiculares con cavidades medulares, dan cuenta del consumo del tuétano. Este recurso alimenticio se caracteriza por ser muy rico en grasas, siendo estas necesarias para hacer frente a las severas condiciones climáticas propias del ambiente de emplazamiento del sitio. Este escenario hace pensar en un uso del espacio acotado en el tiempo, entre los meses de noviembre a marzo donde las condiciones ambientales son más propicias para la instalación humana. Sin embargo, no podemos descartar la ocurrencia de tormentas ocasionales muy fuertes que puedan haber restringido la movilidad de los grupos hasta que cesen. Cabe recordar, que el conjunto óseo está asociado a la presencia de chañar (Geoffroea decorticans), siendo la pulpa de estos frutos comestible y también de elevado valor energético. Por lo tanto, consideramos que se privilegió una ingesta elevada en lípidos para hacer frente a las necesidades metabólicas propias de estos ambientes hostiles (Speth y Spielmann 1983). Asimismo, también podemos relacionar el consumo de la médula con el porcentaje medio-alto de huesos quemados (incipiente y/o avanzado), ya que estos grados de termoalteración podrían relacionarse con el calentamiento del hueso para licuar el tuétano y de esta forma aprovecharlo en su totalidad (Gifford-González 1989).

(d) Un tema interesante que surge también del análisis es la ausencia del consumo de la médula ósea en aquellas falanges que presentaban malformaciones producto posiblemente de alguna enfermedad del animal aprovechado. Esto permite pensar en un tratamiento especial de carácter restrictivo alimentario para aquellas partes de las presas que presentan signos de morbilidad. Al respecto, es significativo que en un contexto ambiental altamente hostil únicamente no fueron aprovechadas esas partes. Sin embargo, esta interpretación queda en el plano hipotético dado lo exiguo de la muestra analizada.

De esta forma, los resultados arqueofaunísticos son totalmente consistentes con aquellos que provienen de otras líneas de evidencia. Al respecto, la combinación de un contexto ecofactual y artefactual discreto, donde en este último se destacan los techos colapsados y la roca con pintura roja, dan cuenta de un uso del espacio acotado y ocasional donde la residencia de corto plazo se relaciona con actividades puntuales relacionadas con aspectos 
ceremoniales desarrolladas por las sociedades formativas (500-700 d.C.). Al respecto, Ratto y Orgaz (2009) interpretan que la combinación del contexto artefactual y su emplazamiento testimonian la celebración de algún tipo de ritual dirigido al volcán Incahuasi, considerándolo de carácter apaciguador por relacionarlo con los eventos catastróficos de origen volcánico que afectaron el bolsón de Fiambalá (Montero et al. 2009). La nueva evidencia aportada por el análisis arqueofaunístico refuerza esta idea.
Agradecimientos: A la memoria de Marcelo Brandán, andinista fallecido en septiembre del año 2010 y que nos acompañó y apoyó en los duros trabajos en terreno realizados en los años 2006 y 2007. A los participantes de las campañas de esos años D. Hershey, A. Feely, D. Carniglia, K. Garrett, y V. Ahumada; como así también el asesoramiento de J. Grant en la temática osteométrica. Por último, a los evaluadores que con sus comentarios han enriquecido al manuscrito.

\section{Referencias Citadas}

Binford, L.R. 1984. Faunal Remains from Klasies River Mouth. Academic Press, Orlando.

Borrero, L.A. 1990. Fuego-Patagonian bone assemblages and the problem of communal guanaco hunting. En Hunters of the Past, editado por L.B. Davis y B.O.K. Reeves, pp. 373-399. Unwin Hyman, Londres.

Bulacio, V. 1998. Desde la Raíz de Catamarca. Publicaciones Legislativas de la Cámara de Senadores, San Fernando del Valle de Catamarca.

Cabrera, A. 1971. Regiones fitogeográficas de la República Argentina. Boletín de la Sociedad Argentina de Botánica 14 (1-2):1-42.

Capaldo, S.D. y R.J. Blumenschine 1994. A quantitative diagnosis of notches made by hammerstone percussion and carnivore gnawing on bovid long bones. American Antiquity 59:724-748.

Cartajena Fasting, I. 2009. Explorando la variabilidad morfométrica del conjunto de camélidos pequeños durante el Arcaico Tardío y el Formativo Temprano en Quebrada Tulán, norte de Chile. Revista del Museo de Antropología 2(1):199-212.

De Nigris, M.E. 2004. El Consumo en Grupos Cazadores Recolectores. Un Ejemplo Zooarqueológico de Patagonia Meridional. Sociedad Argentina de Antropología, Buenos Aires.

Elkin, D.C. 1995. Volume density of South American camelid skeletal parts. International Journal of Osteoarchaeology 5:29-37.

- _ - 1996. Arqueozoología de Quebrada Seca 3: Indicadores de Subsistencia Humana Temprana en la Puna Meridional Argentina. Tesis para optar al grado de Doctor de la Universidad de Buenos Aires.

Gifford-González, D. 1989. Ethnographic analogues for interpreting modified bones: Some cases from East Africa. En Bone Modification, editado por R. Bonnichsen y M. Sorg, pp. 179-246. University of Maine, Orono.

Izeta, A.D., C. Otaola y A. Gasco 2009. Osteometría de falanges proximales de camélidos sudamericanos modernos. Variabilidad, estándares métricos y su importancia como conjunto comparativo para la interpretación de restos hallados en contextos arqueológicos. Revista del Museo de Antropología 2(1):169-180.

Kaufmann, C. 2009. Estructura de Sexo y Edad en Guanaco. Estudios Actualísticos y Arqueológicos en Pampa y Patagonia. Sociedad Argentina de Antropología, Buenos Aires.
Kent, J. 1982. The Domestication and Exploitation of the South American Camelids: Methods of Analysis and their Application to Circum Lacustrine Archaeological Sites in Bolivia and Peru. $\mathrm{Ph}$. D. Dissertation, Washington University, St. Louis.

Kligmann, D. y N. Ratto 2009. Aportes de la sedimentología a la arqueología: el caso del sitio Fiambalá 1 (puna meridional catamarqueña). Actas de Arqueometría Latinoamericana Segundo Congreso Argentino y Primero Latinoamericano, editado por O. Palacios, C. Vázquez, T. Palacios y E. Cabanillas, Vol. 1, pp. 199-208. Comisión Nacional de Energía Atómica, Buenos Aires.

Lyman, R. 1994. Vertebrate Taphonomy. Cambridge University Press, Cambridge.

- _ _ 2008. Quantitative Paleozoology. Cambridge University Press, Nueva York.

Mengoni Goñalons, G.L. 1996. La domesticación de los camélidos sudamericanos y su anatomía económica. En Zooarqueología de Camélidos editado por D.C. Elkin, C.M. Madero, G.L. Mengoni Goñalons, D.E. Olivera, M.C. Reigadas y H.D. Yacobaccio, vol. 2, pp. 33-45. Grupo Zooarqueología de Camélidos, Buenos Aires.

- . - 1999. Cazadores de Guanacos de la Estepa Patagónica. Sociedad Argentina de Antropología, Buenos Aires.

Messineo, P. y C. Kaufmann 2001. Correspondencia de elementos óseos en el sitio Paso Otero 1 (Partido de Necochea, Provincia de Buenos Aires). Intersecciones en Antropología 2:35-45.

Montero López, M.C., F. Hongn, R. Seggiaro, R. Marrett y N. Ratto 2009. Relación entre el volcanismo y los registros arqueológicos en el bolsón de Fiambalá (Departamento Tinogasta, Catamarca). En Entrelazando Ciencias: Sociedad y Ambiente Antes de la Conquista Española, compilado por N. Ratto, pp. 131-158. EUDEBA, Buenos Aires.

Orgaz, M. y F. Morales Morales 1999. Ofrendas Inka en el Cerro Incahuasi. Tinogasta-Argentina. Revista del Proyecto Hombre Andino: Patrimonio e Identidad. Por un Nuevo Amanecer 1(1):13-15. CIUNT, Tucumán.

Ratto, N. 2007. Paisajes arqueológicos en el tiempo: la interrelación de ciencias sociales, físico-químicas y paleoambientales (Dpto. Tinogasta, Catamarca, Argentina). En Producción y Circulación Prehispánicas de Bienes en el Sur Andino, compilado por A. Nielsen, M.C. Rivolta, V. Seldes, M. Vázquez y P. Mercolli, 
Tomo 2, pp. 35-54. Colección Historia Social Precolombina, Editorial Brujas, Córdoba.

Ratto, N., M. Orgaz, G. De La Fuente y R. Plá 2002. Ocupación de pisos de altura y contexto de producción cerámica durante el Formativo: el caso de la región puneña de Chaschuil y su relación con el Bolsón de Fiambalá (Dpto. Tinogasta, Catamarca). Estudios Atacameños 24:51-69.

Ratto, N. y M. Orgaz 2009. Poder, control y volcanes: el Estado Inka en el volcán Incahuasi (Tinogasta, Catamarca, Argentina). En Entrelazando Ciencias: Sociedad y Ambiente Antes de la Conquista Española compilado por N. Ratto, pp. 159-174. EUDEBA, Buenos Aires.

Ratto, N., M.F. Rodríguez y D. Hershey 2010. Explotación y uso de recursos vegetales en sitios arqueológicos del área cordillerana y del valle mesotérmico (Dpto. Tinogasta, Catamarca, Argentina). Actas del XVII Congreso Nacional de Arqueología Chilena, Tomo 2, pp. 1267-1278. Universidad Austral de Chile, Ediciones El Kultrún, Valdivia.
Ratto, N., D. Carniglia y L. Coll 2011. La ocupación de pisos de altura (4000-5000 msnm) por sociedades agropastoriles (ca. 500-700 d.C.) del oeste tinogasteño (Dpto. Tinogasta, Catamarca, Argentina). Manuscrito en posesión de los autores.

Speth J.D. y K.A. Spielmann 1983. Energy source, protein metabolism, and hunter-gatherer subsistence strategies. Journal of Anthropological Archaeology 2:1-31.

Thomas, D.H. y D. Mayer 1983. Behavioral faunal analysis of selected horizons. En The Archaeology of Monitor Valley 2: Gatecliff Shelter, editado por D.H. Thomas, pp. 353-391. Anthropological Papers of the American Museum of Natural History vol 59, parte 1.

Valero-Garcés, B. N. Ratto, A. Moreno, A. Navas y A. DelgadoHuertas 2007. Los Lagos del Altiplano de Atacama y el Noroeste Argentino como Sensores de Cambios Hidrológicos durante el Holoceno. En Escenarios de Cambio Ambiental: Registros del Cuaternario en América Latina editado por M. Caballero. Unión Mexicana de Estudios del Cuaternario (UMEC) y la Universidad Nacional Autónoma de México (UNAM), en prensa.

\section{Nota}

$1 \quad$ Si bien el guanaco patagónico es de mayor tamaño que los guanacos y vicuñas del noroeste argentino debemos tener presente que la anatomía de estas especies es del todo idéntica. Asimismo, las distintas unidades anatómicas se presentan en una escala relativa en la cual la que posee más recursos obtiene el primer lugar y las restantes partes se ubican de acuerdo a la carne y médula que poseen. Por otra parte, hemos preferido la utilización de una especie silvestre como proxy porque todos los indicadores apuntan a señalar que la muestra analizada está conformada por un único individuo cazado (v. gr. vicuña). 